\title{
MiR-221 Promotes Hepatocellular Carcinoma Cells Migration via Targeting PHF2
}

\author{
Yi Fu (D, ${ }^{1}$ Mingyan Liu, ${ }^{2}$ Fengxia Li, ${ }^{2}$ Li Qian, ${ }^{2}$ Ping Zhang, ${ }^{3}$ Fengwei Lv, \\ Wenting Cheng, ${ }^{2}$ and Ruixing Hou $\mathbb{D}^{3}$ \\ ${ }^{1}$ Department of Human Anatomy, Histology and Embryology, School of Biology and Basic Medical Sciences, \\ Soochow University, Suzhou 215007, China \\ ${ }^{2}$ School of Medicine, Yangzhou University, Yangzhou 225001, China \\ ${ }^{3}$ Institute of Hand Surgery, Ruihua Affiliated Hospital of Soochow University, Suzhou 215007, China
}

Correspondence should be addressed to Yi Fu; yfu@suda.edu.cn

Received 17 January 2019; Revised 4 April 2019; Accepted 14 April 2019; Published 12 May 2019

Academic Editor: Fumio Imazeki

Copyright (C) 2019 Yi Fu et al. This is an open access article distributed under the Creative Commons Attribution License, which permits unrestricted use, distribution, and reproduction in any medium, provided the original work is properly cited.

\begin{abstract}
MicroRNAs (MiRNAs), which regulate the gene expression leading to translational inhibition or mRNA degradation, are involved in carcinogenesis and tumor progression. Previous studies have demonstrated that miR-221 was one of the most consistent overexpressed miRNAs in several types of cancer. However, the role of miR-221 in human liver cancer progression is not yet fully elucidated. Levels of miR-221 and plant homeodomain finger 2 (PHF2) expressions in human hepatocellular carcinoma (HCC) tissues and cell lines were detected using western blotting and quantitative real-time PCR (qRT-PCR). Cell migration was studied using the transwell assays. A dual-luciferase reporter system was used to validate the target gene of miR-221. The results indicated that miR-221 promoted HCC cell migration. By performing subsequent systematic bioinformatic analyses, we found PHF2 was the target gene of miR-221 and the direct binding relationship was further validated by dual-luciferase reporter assay. In addition, lower expression of PHF2 promoted HCC cell migration and linked to worse overall survival in HCC patients. Finally, the negative correlation between miR-221 and PHF2 expression levels in HCC specimens was further confirmed. Taken together, our findings implied that miR-221 could be a potential candidate for the therapeutics of HCC metastasis.
\end{abstract}

\section{Introduction}

Hepatocellular carcinoma (HCC) is the fifth common cancer and third leading cause of cancer-related deaths worldwide, and it is considered to be one of the most common cancers with poor prognosis [1]. Due to the rapid development of sequencing technology, there is a growing comprehension on the molecular mechanisms resulting in HCC carcinogenesis. Previous studies have considerably focused on study of DNA mutations and gene expression changes in HCC [2]. Moreover, RNA mutations and change of mRNA transcription are also correlated with the initiation and progression of HCC.

MicroRNAs (miRNAs) are small noncoding RNAs with approximately 22 nucleotides that could play important regulatory roles in plants and animals by targeting mRNAs for translational suppression [3]. In excess of 2,000 miRNAs have been identified to regulate a variety of protein coding transcription [4]. By modulating gene expression via posttranscriptional mechanisms, miRNAs are now known as vital players in cell cycle, differentiation, apoptosis, and oncogenesis [5]. Furthermore, miRNAs are identified to be correlated with the regulation of epithelial-mesenchymal transition and tumor metastasis by targeting important genes $[6,7]$. MiR-221, which is encoded by human chromosome Xp11.3, is often abnormally expressed and associated with the regulation of oncogenes or tumor suppressive genes. Among numerous miRNAs, the upregulation of miR-221 has been recently recognized in numerous types of human cancers $[8,9]$. Thus, identification of the function of miR-221 and its targets could make a new access to cancer treatment.

The aim of our study was to investigate miR-221 expression in HCC cells and tissues and to observe the changes in the migration ability of HCC cells following variation of the miR-221 expression. Our data, for the first time, revealed the 
tumorigenesis role of miR-221 in HCC and identified a target gene plant homeodomain finger 2 (PHF2). PHF2, which maps to human chromosome (Chr) 9q22 [10], is a member of the KDM7 histone demethylase family that contains a plant homeodomain (PHD) in the Jumonji-C and N-terminal domain [11]. Notably, previous studies indicated that PHF2 acts as a cancer suppressor by regulating p53 in colon cancer tissues [12]. However, the role of PHF2 in HCC remains to be investigated. In our study, we demonstrated that miR-221 promoted HCC cells migration via targeting PHF 2 and could be a new target for HCC therapeutics. Taken together, our results may provide critical strategy for targeted therapy and prognosis of HCC.

\section{Materials and Methods}

2.1. Patients and Specimens. 60 patients with hepatocellular carcinoma who underwent resection were collected from Affiliated Hospital of Yangzhou University between 2014 and 2017. The tissue microarray (TMA) consisted of 60 surgical cases produced by the National Engineering Centre for Biochip (Shanghai, China). The patients' clinicopathologic parameters including tumor diameter, sex, age, TNM stage, lymph node metastasis, and depth of invasion. These data were acquired from the Medical Record of the Affiliated Hospital of Yangzhou University. 3-year clinical follow-up data were obtainable for 60 patients from the Yangzhou area. The median follow-up time is 20 months. And the cases of TMA include 2 lost follow-up patients. All the tissues were collected for the present study with patients' informed consent. And the study of human specimens was approved by the Review Board of the Affiliated Hospital of Yangzhou University.

2.2. Tissue Samples. 36 patients with histologically conformed hepatocellular carcinoma tissues (HCT) were obtained from the first Affiliated Hospital of Yangzhou University. The cancer tissues and adjacent cancerous tissues were collected from patients. All samples were acquired at the time of surgery and were frozen in liquid nitrogen immediately. This investigation was approved by the medical ethics committee of Yangzhou University and informed consent was gained from patients before recruitment.

2.3. Immunohistochemistry. Immunohistochemistry was performed with a standard avidin biotinylated-HRP complex (ABC) kit (Zhongshan biotech, Beijing, China) following the $A B C$ method. The slips were incubated with antiPHF2 antibody (1:1000) (abcam) overnight at $4^{\circ} \mathrm{C}$, and diaminobenzidine (DAB; Zhongshan Biotech, Beijing, China) was used to turn out a brown precipitation. The immunoreactivity was evaluated blindly by three pathologists using light microscopy (Olympus BX-51 light microscope), and the image was collected by Camedia Master C-3040 digital camera. The level of PHF2 was ranked as high when $\geqq 5 \%$ of tumor cells showed immunopositivity. Biopsies with $<5 \%$ tumor cells immunostaining were regarded as low.

2.4. Cell Lines and Culture. HCC cell lines SMMC-7721, Bel7402, MHCC97, and HepG2 cells were obtained from the
American Type Culture Collection (ATCC). Human normal hepatocyte HL-7702 cells were also obtained from the ATCC. HL-7702, SMMC-7721, and Bel-7402 cells were cultured in RPMI-1640 (GIBCO, US). HepG2 and MHCC97 cells were cultured in DMEM (GIBCO, US). The culture media were in humidified air with $5 \% \mathrm{CO} 2$ at $37^{\circ} \mathrm{C}$, supplemented with $10 \%$ fetal bovine serum and $1 \%$ streptomycin/penicillin.

2.5. Western Blot. After transfection, cells were harvested from the plates. Equivalent proteins were separated by $10 \%$ SDS polyacrylamide gel electrophoresis (SDS-PAGE); the proteins were then transferred onto PVDF membranes. After incubated overnight at $4^{\circ} \mathrm{C}$ with appropriate primary antibodies, the membranes were further probed with a horseradish peroxidase-conjugated secondary antibody (1:2000) for $2 \mathrm{~h}$ at room temperature. The membrane was detected by enhanced chemiluminescence (ECL) solution and scanned on the chemiluminescence imaging analysis system (Tanon Biotechnology, Shanghai, China). Each western blot was repeated three times.

2.6. RNA Extraction and Quantitative Real-Time PCR ( $q R T$ $P C R$ ). After cell transfection, the cellular RNA was extracted from cell lines or tissues using TRIzol reagent (Sigma). Then the RNA transcribed into cDNA by PrimeScript RT master Mix (Takara, Dalian, China) following the corresponding protocols. qRT-PCR was carried out with a SYBR GREEN MIX kit (Promega, Madison, USA) conforming to the manufacturer's instructions. The qRT-PCR detection was carried out using the ABI 7500 FAST Real-Time PCR System. GAPDH was used for loading control. The relative level was calculated using the relative quantification equation (RQ) = $2^{-\Delta \Delta \mathrm{Ct}}$

2.7. Wound Healing Assays. SMMC-7721 cells were grown to $80 \%$ proportion in 6 -well plates wounded by scratching the cell monolayer with a sterilized $200 \mu$ l pipette tip. Phase contrast images were collected in the same field at indicated time periods $(0,24$, and 48 hours) using the Nikon Digital Microscope with magnification of $\times 100$. Experiments were performed in thrice.

2.8. Cell Migration Assays. Cell migration assays were performed by modified two-chamber plates with a pore size of $8 \mu \mathrm{m}$. Cells were seeded in serum-free medium in the upper chamber at a density of $1 \times 10^{5}$ cells/well. After 24 $h$ incubation in $37^{\circ} \mathrm{C}$, the cells were fixed in methanol and stained with trypan blue. Cells in the upper chamber were carefully removed with a cotton swab and the cells that traversed the membrane were counted under a microscope in five fields. The analysis was performed thrice.

2.9. Dual-Luciferase Report Assay. MiRNA-binding regions of PHF2 for miR-221-3p in the $3^{\prime}$-UTR were subcloned into the GP-miRGLO luciferase miRNA vector. SMMC-7721 cells were seeded in 24 -well plates at a density of $5 \times 10^{4}$ cells per well and transfected with wild type or mutant luciferase reporter plasmids at the concentration of $50 \mathrm{nmol} / \mathrm{L}$. After 24 hours of incubation, luciferase activity was measured by 
TABLE 1: Correlation between the HCC patients' clinicopathologic characteristics and miR-211 expression.

\begin{tabular}{|c|c|c|c|}
\hline \multirow[b]{2}{*}{ Variables } & \multicolumn{3}{|c|}{ miR-221 expression } \\
\hline & $\mathrm{n}=36$ & $2^{-\Delta \Delta C t}$ & $P *$ \\
\hline \multicolumn{4}{|l|}{ Age } \\
\hline$\leq 57$ years & 13 & $2.63 \pm 0.54$ & \multirow[t]{2}{*}{0.340} \\
\hline$>57$ years & 23 & $2.85 \pm 0.50$ & \\
\hline \multicolumn{4}{|l|}{ Gender } \\
\hline Male & 26 & $2.64 \pm 0.75$ & \multirow[t]{2}{*}{0.415} \\
\hline Female & 10 & $2.48 \pm 0.33$ & \\
\hline \multicolumn{4}{|l|}{ Tumor size } \\
\hline$\leq 7 \mathrm{~cm}$ & 20 & $2.33 \pm 0.37$ & \multirow[t]{2}{*}{0.001} \\
\hline$>7 \mathrm{~cm}$ & 16 & $3.31 \pm 0.23$ & \\
\hline \multicolumn{4}{|l|}{ pT status } \\
\hline $\mathrm{pT}_{1-2}$ & 21 & $2.68 \pm 0.52$ & \multirow[t]{2}{*}{0.161} \\
\hline $\mathrm{pT}_{3-4}$ & 15 & $2.98 \pm 0.59$ & \\
\hline \multicolumn{4}{|l|}{ pN status } \\
\hline $\mathrm{pN}_{0-1}$ & 12 & $2.37 \pm 0.40$ & \multirow[t]{2}{*}{0.001} \\
\hline $\mathrm{pN}_{2}-\mathrm{pN}_{3}$ & 24 & $3.28 \pm 0.21$ & \\
\hline \multicolumn{4}{|l|}{ TNM stage } \\
\hline II & 18 & $2.34 \pm 0.37$ & \multirow[t]{2}{*}{0.001} \\
\hline III-IV & 18 & $3.28 \pm 0.24$ & \\
\hline \multicolumn{4}{|l|}{ Serum AFP } \\
\hline$\leq 400 \mu \mathrm{g} / \mathrm{L}$ & 11 & $2.40 \pm 0.47$ & \multirow[t]{2}{*}{0.014} \\
\hline$>400 \mu \mathrm{g} / \mathrm{L}$ & 25 & $2.55 \pm 0.47$ & \\
\hline \multicolumn{4}{|c|}{ Microvascular invasion } \\
\hline Yes & 10 & $2.81 \pm 0.62$ & \multirow[t]{2}{*}{0.268} \\
\hline No & 26 & $3.09 \pm 0.61$ & \\
\hline \multicolumn{4}{|c|}{ Portal vein tumor thrombus } \\
\hline Yes & 9 & $2.87 \pm 0.30$ & \multirow[t]{2}{*}{0.323} \\
\hline No & 27 & $3.14 \pm 0.43$ & \\
\hline
\end{tabular}

a dual-luciferase reporter system (Promega, Fitchburg, WI, USA).

2.10. Statistical Analysis. Statistical analysis was executed by SPSS 16.0 software and images were obtained with GraphPad Prism 5 Software. The grayscale detection software is Image J. The data are shown as the mean \pm standard deviation (SD). The correlation analyses were using Pearson's correlation analyses, and the between-group differences were evaluated using Student's T test or one-way ANOVA. For TMA, the relationship between PHF2 and the clinicopathologic factors of the HCC patients was evaluated by $\chi 2$ test. The KaplanMeier method and log-rank test were employed to assess the correlation between PHF2 expression and patient survival. $\mathrm{P}<0.05$ is identified as statistically significant $(* \mathrm{P}<0.05$, $* * P<0.01, * * * \mathrm{P}<0.001)$.

\section{Results}

3.1. MiR-221 Expression Is Increased in HCC Cell Lines and Tissues. To investigate the mRNA level of miR-221 in HCC cell lines, qRT-PCR was performed and results demonstrated that the miR-221 mRNA levels in HCC cell lines were higher than human normal hepatocyte (HL-7702) (Figure 1(a)). Moreover, we also found that miR-221 levels were higher in HCC tissues $(\mathrm{T})$ than in adjacent noncancerous tissues $(\mathrm{N})(\mathrm{n}=36$, Figure 1(b), Supplemental Figure 1). Results demonstrated that higher miR-221 expression was evidently associated with HCC. Thus, we used HCC cells to examine the role of miR-221 on cell migration. Furthermore, we demonstrated the correlation of the relative expression of miR-221 with the clinicopathological features of HCC patients in Table 1. Results showed that miR-221 expression in HCC patients with tumor size $(\leq 7 \mathrm{~cm}), \mathrm{pN}$ status $\left(\mathrm{pN}_{0-1}\right)$ or TNM stage II was evidently lower than that with tumor size $(>7 \mathrm{~cm}), \mathrm{pN}_{2}-\mathrm{pN}_{3}$ or TNM stage III-IV $(\mathrm{p}<0.05)$. The relative expression of miR-221 was not found to be associated with age, gender, pT status, or serum AFP levels of HCC patients ( $\mathrm{p}>0.05)$. Therefore, miR-221 expression is increased in HCC tissues and had an evident relationship with the HCC patients' characteristics.

3.2. MiR-221 Promotes the HCC Cell Lines Migration. To validate whether miR-221 was associated with the migration of HCC cells, we examine the effect of miR-221 on the cell wound healing. We found that miR-221-transfected cells 


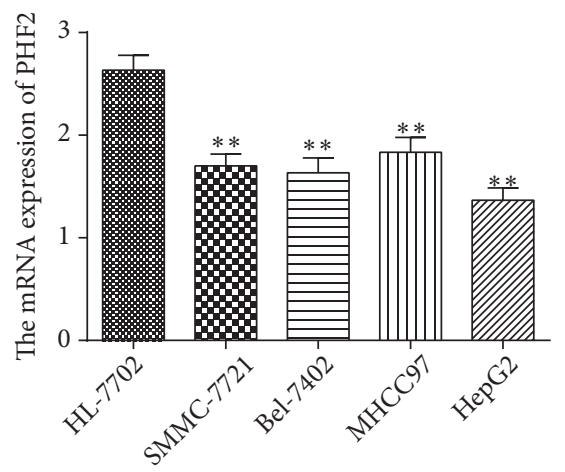

(a)

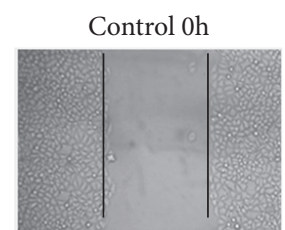

Control 24h

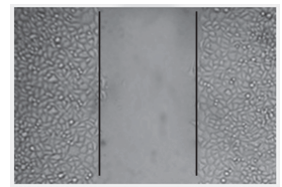

Control 48h
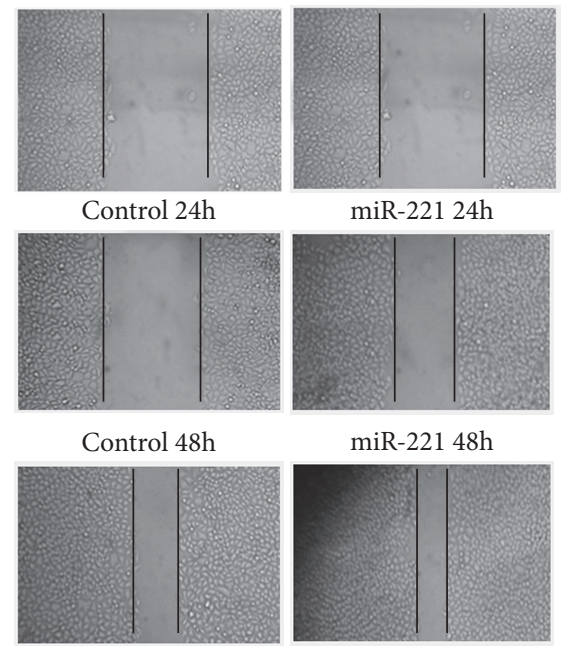

miR-221 24h

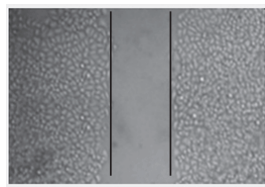

miR-221 48h

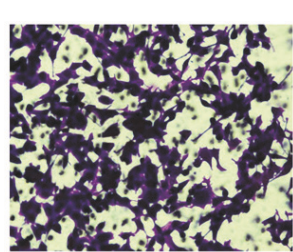

Control

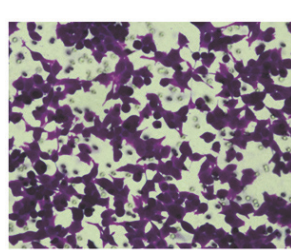

control
miR-221
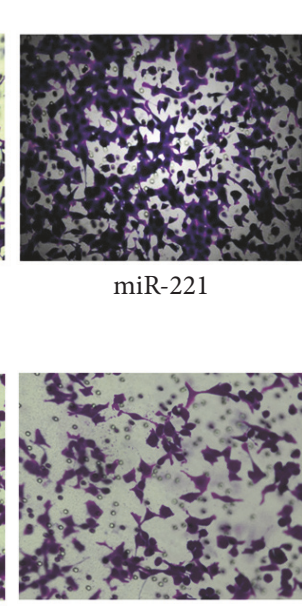

anti-miR-221 (c)

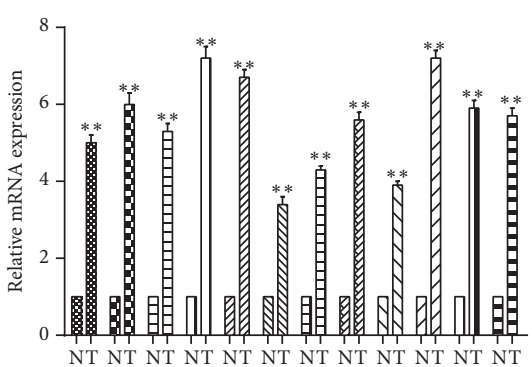

(b)

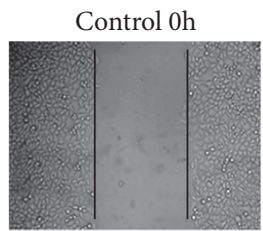

Control 24h

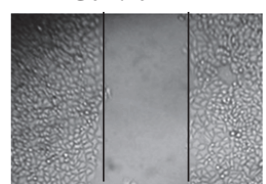

Control 48h

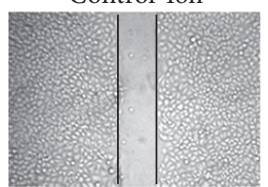

anti-miR-221 48h

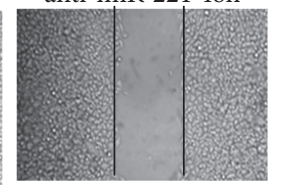

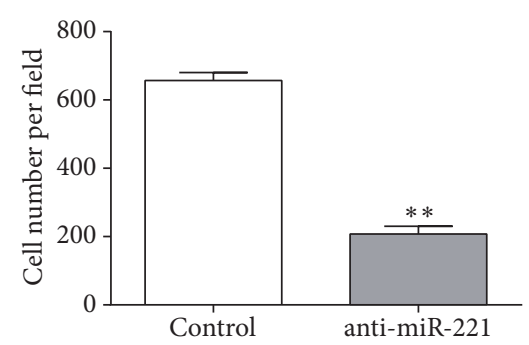

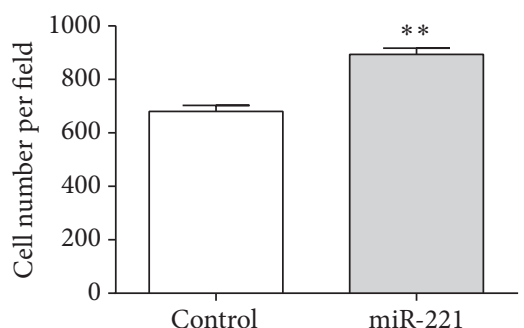

(d)

FIGURE 1: miR-221 is upregulated in HCC tissues and cell lines and promotes the cell migration. (a) qRT-PCR results of miR-221 mRNA levels in HCC cell lines and human normal hepatocyte. (b) Quantitative PCR results of miR-221 mRNA levels in HCC tissues (T) and in adjacent noncancerous tissues $(\mathrm{N})$. MiR-221 mRNA levels are higher in the HCC tissues $(\mathrm{T})$ than in adjacent noncancerous tissues $(\mathrm{N})(\mathrm{n}=12)$. (c) miR-221 increases SMMC-7721 cells wound healing. Anti-miR-221 inhibits SMMC-7721 cells wound healing. Lines indicate the border of the healing wounds. (d) The cell migration of SMMC-7721 cells after transfection with miR-221 or anti-miR-221 and their negative control detected by transwell assays. Each bar represents the mean $\pm \mathrm{SD}$ of three independent experiments. $* * P<0.01$. 
showed a longer distance of shift whereas anti-miR-221transfected groups showed shorter shift when compared with relevant negative control group (Figure $1(\mathrm{c})$ ). The data showed that miR-221 promoted HCC cells wound healing capability. We then investigated the effect of miR-221 on HCC cell lines migration and found that miR-221-transfected SMMC-7721 cells enhanced the number of cells penetrating the inserts. In contrast, miR-221 inhibition decreased the abilities of SMMC-7721 cells to penetrate the inserts (Figure $1(\mathrm{~d})$ ). The results revealed that miR-221 could promote cell migration in SMMC-7721 cells.

3.3. MiR-221 Influences Metastasis-Related Genes. To investigate the effect of miR-221 on cell migration, we carried molecular analyses to detect the expression of some type metastasis-related genes. Our results showed that miR-221 downregulated the expression of an epithelial marker (Ecadherin) in mRNA levels in SMMC-7721 cells, whereas antimiR-221 led to the opposite results (Figure 2(a)). Meanwhile, the mRNA level of a mesenchymal marker (N-cadherin) was increased when the HCC cells was transfected with miR-221. And anti-miR-221 decreased the expression of $\mathrm{N}$-cadherin in mRNA levels (Figure 2(b)). Furthermore, miR-221 had the same effect on E-cadherin and $\mathrm{N}$-cadherin in protein levels (Figures 2(c) and 2(d)). It is identified that the epithelialto-mesenchymal transition (EMT) transcription factors play a crucial role in the process of EMT of cancer cells. We performed western blot to verify which EMT transcription factors miR-221 regulates. And the results demonstrated that miR-221 positively regulated the EMT transcription factors Snail and Slug (supplemental Figure 2). These data suggested that miR-221 significantly influenced the expression of EMT transcription factors and biomarkers. Taken together, miR221 has a crucial impact on HCC cell migration.

3.4. PHF2 Is a Target Gene of miR-221. The mechanism of the migration regulated by $\mathrm{miR}-221$ has not been well indicated. Then we found putative genes that miR-221 might regulate by bioinformatics systems. Bioinformatics analysis was performed in two online predicting algorithms miRDB (http://www.mirdb.org/) and TargetScan (http://www.targetscan.org/) to identify miR-221 target genes. Among these genes PHF2 gene was our applicant target. The results showed that miR-221 remarkably reduced the protein PHF2 expression in SMMC-7721 cells. Conversely, anti-miR-221 significantly increased the protein level of PHF2 (Figure 3(a)). In $\mathrm{qPCR}$, our data indicated that miR-221 downregulated PHF2 mRNA levels in SMMC-7721 cells, whereas antimiR-221 led to the opposite results (Figure 3(b)). To verify whether PHF2 is a target gene of miR-221, we established the dual-luciferase reporter vectors containing mutation type (Mut) or wild type (WT) fragments of PHF2 $3^{\prime}$-UTR. The dual-luciferase reporter assay results showed that miR-221 suppressed the activity of luciferase in WT-transfected HCC cells (Figure 3(c)). These results demonstrated the PHF2 $3^{\prime}$ UTR is a target of miR-221 in HCC cells.

3.5. PHF2 Inhibits HCC Cell Migration. To investigate the role of PHF2 in HCC cell lines, transwell analyses were used to examine the cell migration. The data showed that knockdown of PHF2 increased the number of migratory cells in contrast to negative control group. Meanwhile, following overexpression of PHF2 transfection, the number of migratory cells was decreased compared to negative control group (Figure 3(d)). Thus, PHF2 could inhibit the cell migration of HCC cells. For that PHF2 is a direct target of miR-221 in HCC cells, this is supported by our previous study that miR-221 promoted the migration of HCC cells and anti-miR-221 suppressed the migration of HCC cells in vitro. To investigate the role of miR221-PHF2 pathway in HCC tumorigenesis, we performed the restoration of PHF2 in miR-221 overexpression cells. Notably, PHF2 rescued miR-221 mediated promotion of migration in SMMC-7721 cells (Figure 3(e)). Collectively, these data indicated that miR-221 could promote cell migration of HCC cells by downregulating PHF2.

3.6. The Role of miR-221 and PHF2 Expression in Human HCC. We performed immunohistochemistry staining of TMA slide containing HCC/adjacent cancerous tissues and found that PHF2 protein was situated in the cytoplasmic (Figure 4(a)). In adjacent cancerous tissues, high PHF2 staining was recorded in $66.6 \%$ (40 of 60 cases). In HCC tissues, high expression of PHF2 was observed in 38.3\% (23 of 60 cases). Higher expression of PHF2 was detected in adjacent cancerous tissues compared to the carcinoma tissues $(P<$ 0.05 , paired $\chi 2$ test). TNM stage is the most important prognostic indicator for HCC patients, so we investigated whether the protein level of PHF2 was correlated with TNM stage. Our results showed that PHF2 staining was increased in TNM stages II compared with stages III-IV $(\mathrm{P}<0.05$, paired $\chi 2$ test) (Figure 4(b)). Furthermore, the protein level of PHF2 was also correlated with lymph node metastasis-pN status, depth of invasion and serum AFP $(\mathrm{P}<0.05$, paired $\chi 2$ test) (Table 2). Nevertheless, we did not find the correlation between PHF2 and other clinicopathologic factors including tumor size, age, gender, microvascular invasion, and portal vein tumor thrombus. Overall survival was used for survival analysis and the overall mortality events were 50 . The period of the follow-up is 36 months. Kaplan-Meier survival analysis illustrated a higher overall survival in HCC patients with high PHF2 expression than those with low PHF2 expression $(\mathrm{P}=$ 0.0437, log-rank test) (Figure 4(c)). To investigate the miR221 and PHF2 protein levels in vivo, 12 human HCC tissues were detected by the qRT-PCR. The results indicated a negative correlation between the expressions of PHF2 and miR221 (Figure 4(d)). Furthermore, we performed qRT-PCR and results showed that the PHF2 mRNA levels in HCC cell lines were lower than human normal hepatocyte (HL-7702) (supplemental Figure 4). These suggested that PHF2 was negatively correlated with miR-221 and had an evident relationship with clinicopathological parameters in HCC tissues.

\section{Discussion}

HCC has the highest mortality as a primary cancer for its strong malignant proliferation and migration [13]. The advance diagnosis of HCC largely improves the therapeutic efficacy in patients, thereupon, a sensitive and specific 

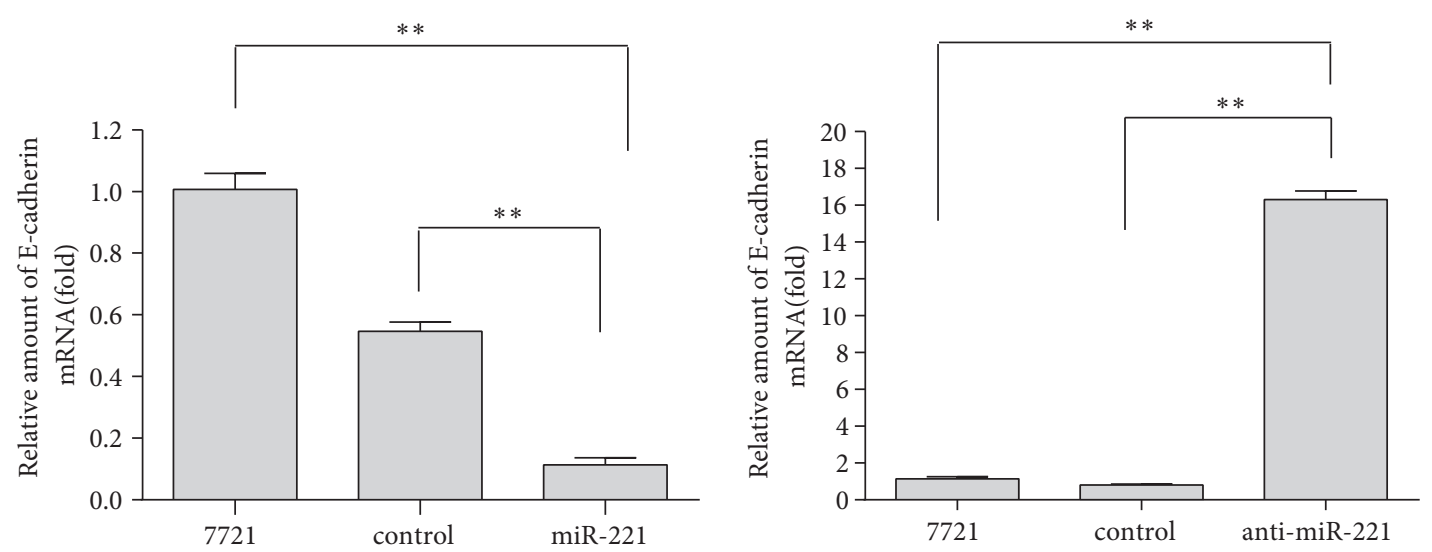

(a)
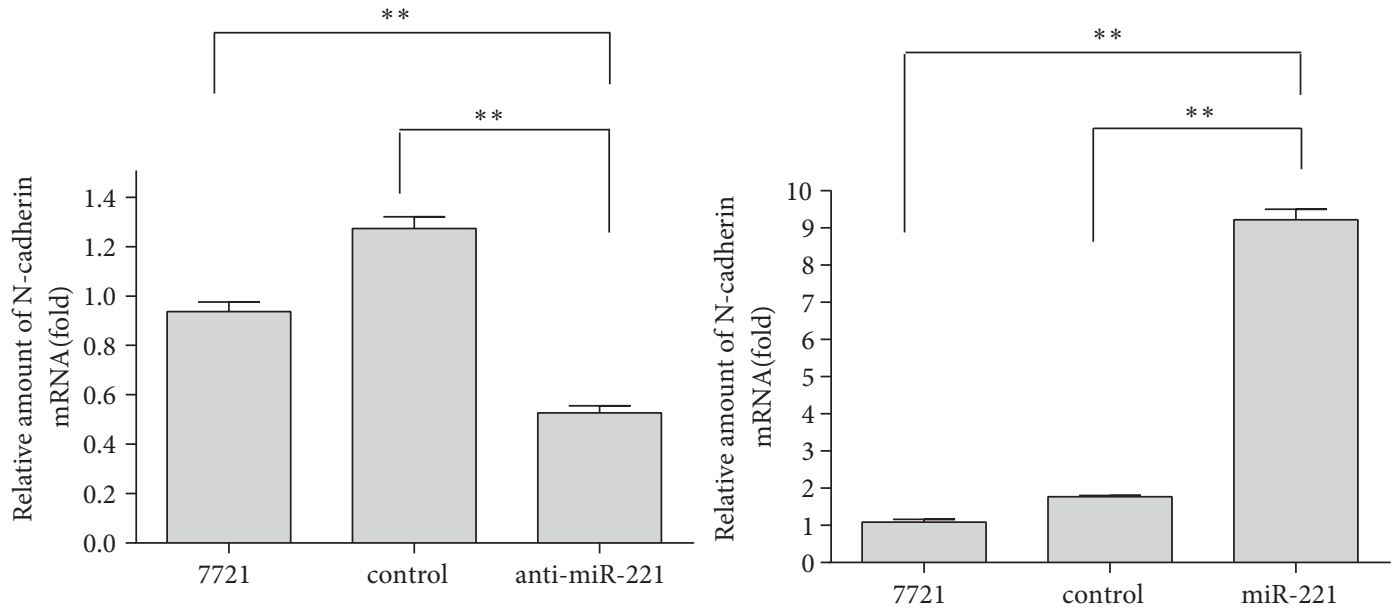

(b)
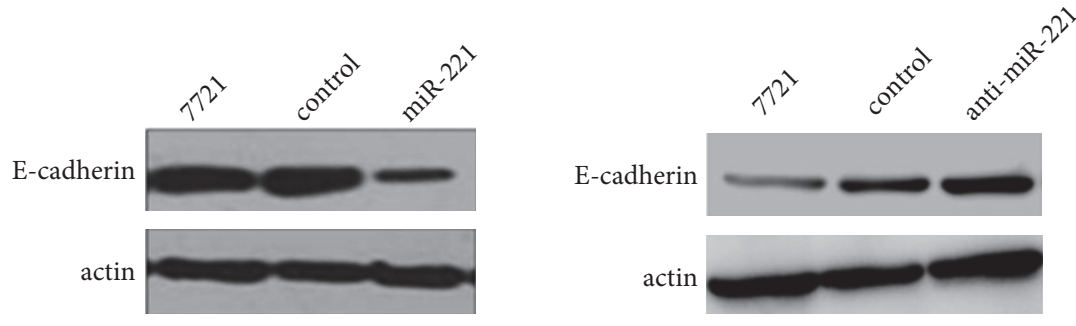

(c)
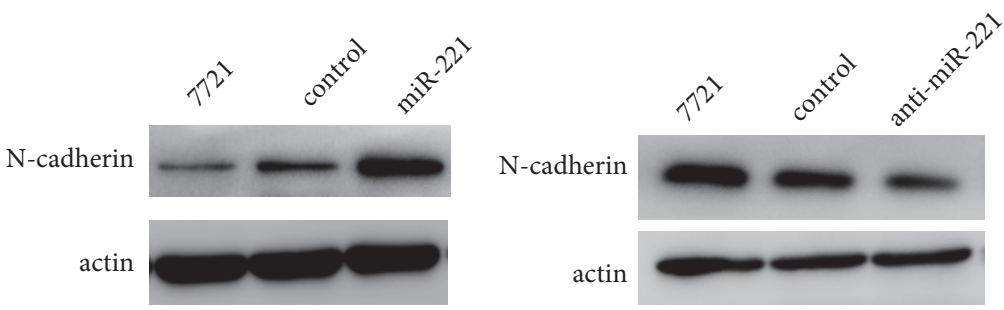

(d)

FIGURE 2: MiR-221 has effects on some key metastasis-related proteins. (a-b) qRT-PCR was used to detect E-cadherin and N-cadherin mRNA level in miR-221-overexpression and miR-221-knockdown SMMC-7721 cells. (c-d) Western blot was used to detect E-cadherin and N-cadherin protein level in miR-221-overexpression and miR-221-knockdown SMMC-7721 cells. Each bar represents the mean \pm SD of three independent experiments. $* * P<0.01$. 


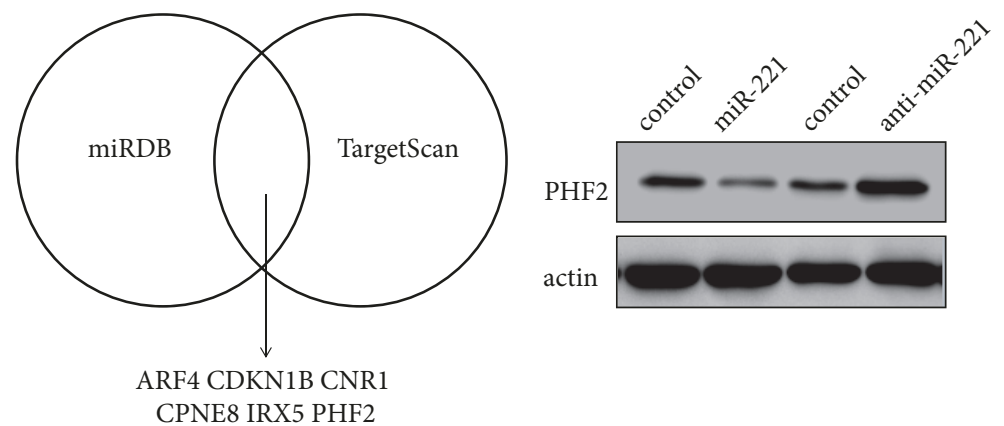

(a)
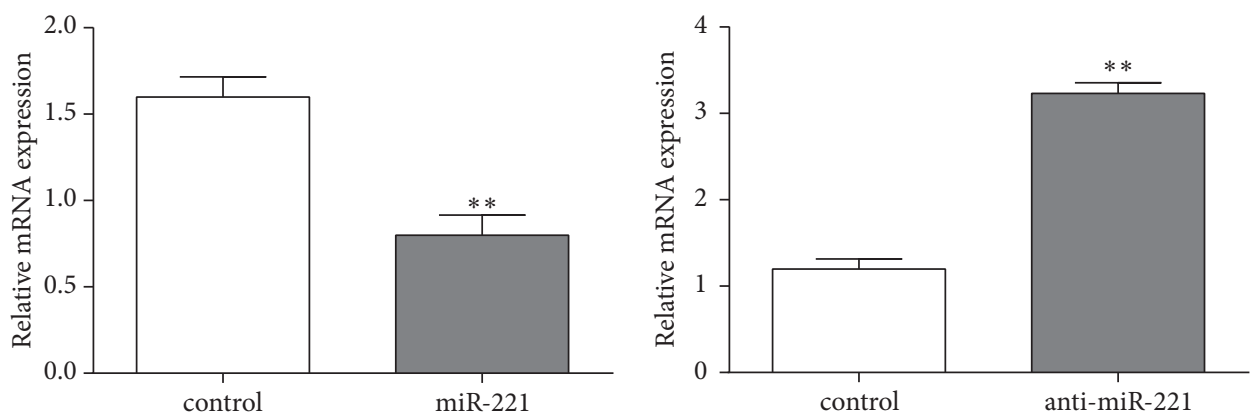

(b)

miR-221-3p 3' CUUUGGGUCGUCUGUUACAUCGA $5^{\prime}$<smiles></smiles>

PHF2 WT 5' CAUAUUUUUCAUAGAAUGUAGCG 3

PHF2 Mut 5' CAUAUUUUUCAUAGATACATCGA $3{ }^{\prime}$

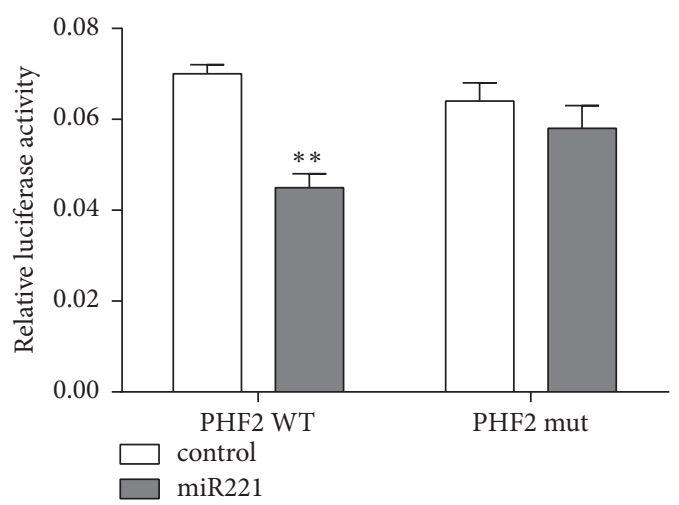

(c)

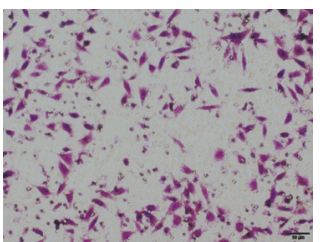

si-Control

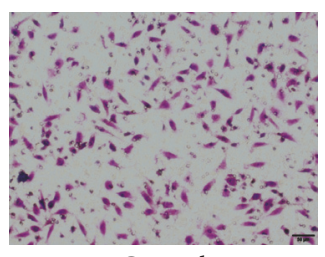

Control

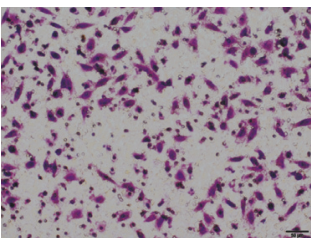

si-PHF2

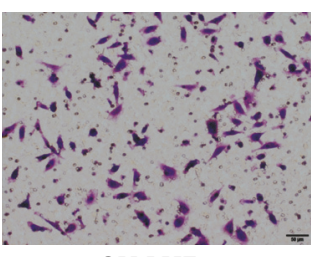

OV-PHF2
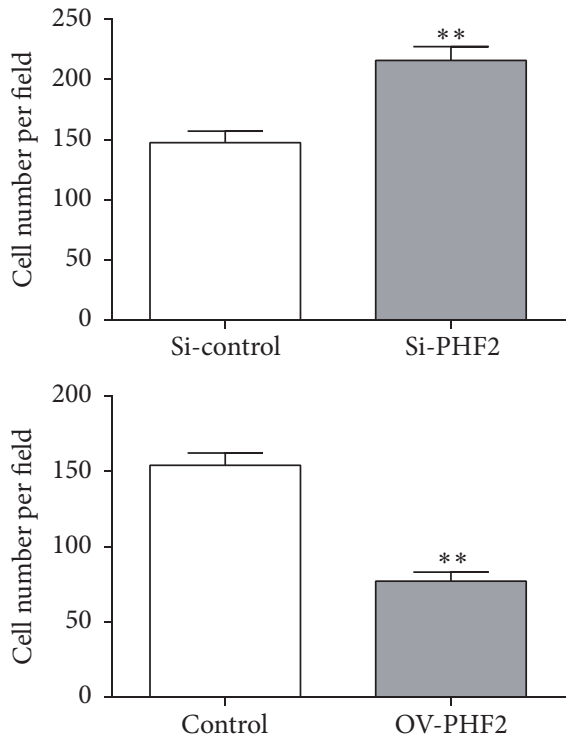

(d)

Figure 3: Continued. 


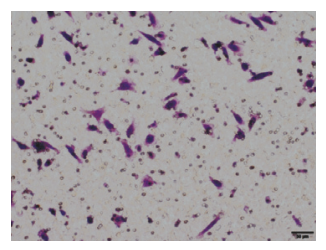

Control

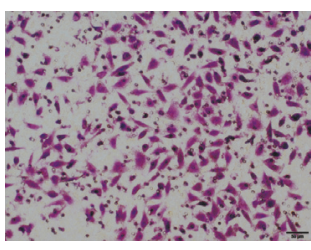

$\operatorname{miR}-221$

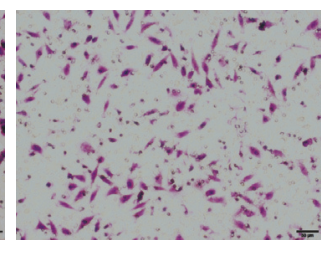

miR-221+OV-PHF2 (e)

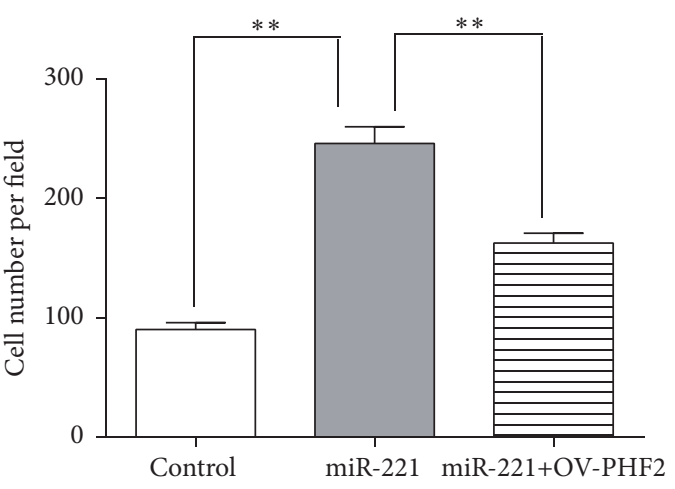

FIgURE 3: PHF2 is a direct target of miR-221. (a) miRNAs were computationally predicted using two independent miRNA databases. The PHF2 protein level was measured by western blot analysis in SMMC-7721 cells transfected with miR-221 or anti-miR-221. (b) qRT-PCR results of PHF2 mRNA levels after transfecting SMMC-7721 cells with miR-221 or anti-miR-221. (c) Schematic description of wild type (WT) and mutated $3^{\prime}$-UTR of the PHF2 mRNA. The WT and mutated $3^{\prime}$ UTR sequences were inserted into luciferase reporter plasmids. In luciferase activity assays, miR-221 suppressed luciferase activity of the wild type but not mutant PHF2 $3^{\prime}$-UTR constructs in SMMC-7721 cells. (d) Knockdown of PHF2 promotes the migration of SMMC-7721 cells. Overexpression of PHF2 inhibits the migration of SMMC-7721 cells. (e) Restoration of PHF2 rescues miR-221 mediated promotion of cell migration. Each bar represents the mean \pm SD of three independent experiments. $* * P<0.01$.

marker is extremely critical [14]. Previous research identified biomarkers mainly focused on proteins [15]; however, miRNAs have absorbed the attention from investigators for the low cost of validation as new molecular markers [16]. Furthermore, dysregulation of miRNAs is a general incident that influences cell invasion, migration, apoptosis, and proliferation in tumor progression [17]. The aim of our study is to investigate the role of miR-221 in HCC and the candidate as diagnostic and therapeutic indicator. Our study demonstrated that the level of miR-221 in HCC is higher than that in adjacent cancerous tissues and cell lines. These indicated that miR-221 could be regarded as a biomarker in early diagnosis and thereby establishing new treatment strategies for HCC.

MiR-221 has been identified to be abnormally regulated in various tumors and involved in cancer cell proliferation and EMT transition in breast cancers [18-21]. Hence, we explored the biological role of miR-221 in HCC cell lines, and our results demonstrate that miR-221 could evidently promote HCC cell migration, while inhibition of miR-221 suppressed the cell migration. It is identified that epithelialto-mesenchymal transition (EMT) which could induce stem cell features was accompanied by a stable increase in EMTassociated mesenchymal markers and a decrease in epithelial markers $[22,23]$. Previous studies have showed that miR221 increased E-cadherin level in an EMT-induced cell line [23] and miR-221 was downregulated by EMT transcription factor Slug in human breast cancer cells [19]. However, the correlation of miR-221 and $\mathrm{N}$-cadherin in HCC remains to be clarified. In our study, the mRNA and protein level of $\mathrm{N}$ cadherin was increased when the HCC cells were transfected with miR-221. Meanwhile, miR-221 could downregulate the E-cadherin expression in protein and mRNA levels. Although miR-221 could regulate the protein level of E-cadherin, Nassirpour et al. were unable to identify the matching sequence in the $3^{\prime}$ UTR [24]. Therefore, E-cadherin could not be a target gene of miR-221. Then we determined the potential target genes of miR-221 with miRDB databases and TargetScan. The genes calculated by algorithms were selected as potential genes of miR-221. PHF2 is found to be most promising among the candidates.

Recently, PHF2 has been shown to act as a tumor repressor associated with p53 in colon and stomach tumor development $[12,25]$. Meanwhile, PHF2 are overexpressed in esophageal squamous cell carcinoma (ESCC) and was associated with decreased overall survival of ESCC patients [26]. However, association of PHF2 with the underlying molecular mechanisms in HCC cells is poorly understood. In the current study, our findings showed a negative correlation between PHF2 and miR-221. MiR-221 decreased the expression of PHF2 in both mRNA and protein levels. As shown in luciferase reporter assay, miR-221 inhibited the activity of luciferase in WT-PHF2-3'-UTR transfected HCC cells. These demonstrated that PHF2 $3^{\prime}$-UTR was a target of miR-221-3p in HCC cells. We also found PHF2 inhibited HCC cell migration and an evident lower expression of PHF2 was detected in the carcinoma tissues compared with adjacent cancerous tissues. Meanwhile, the protein level of PHF2 was correlated with depth of invasion, TNM stage and lymph node metastasis-pN status. Furthermore, we verified the negative correlation between the mRNA level of miR-221 and PHF2 in clinical HCC patients by Pearson's correlation coefficient analysis. To the best of our knowledge, this is the first study that investigated the role of PHF2 as a target gene of miR-221 in HCC development.

Taken together, this study showed that miR-221 was upregulated in HCC cells and tissues and revealed the tumorigenesis role in HCC cells. Moreover, we also identified PHF2 as a target gene of miR-221 in experimental and clinical levels. Our data indicated that miR-221 participated in HCC 


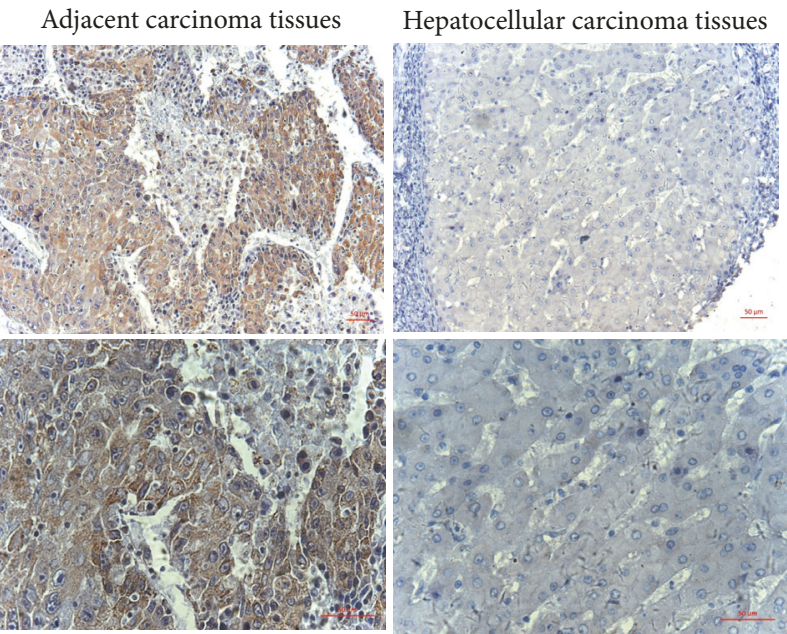

(a)
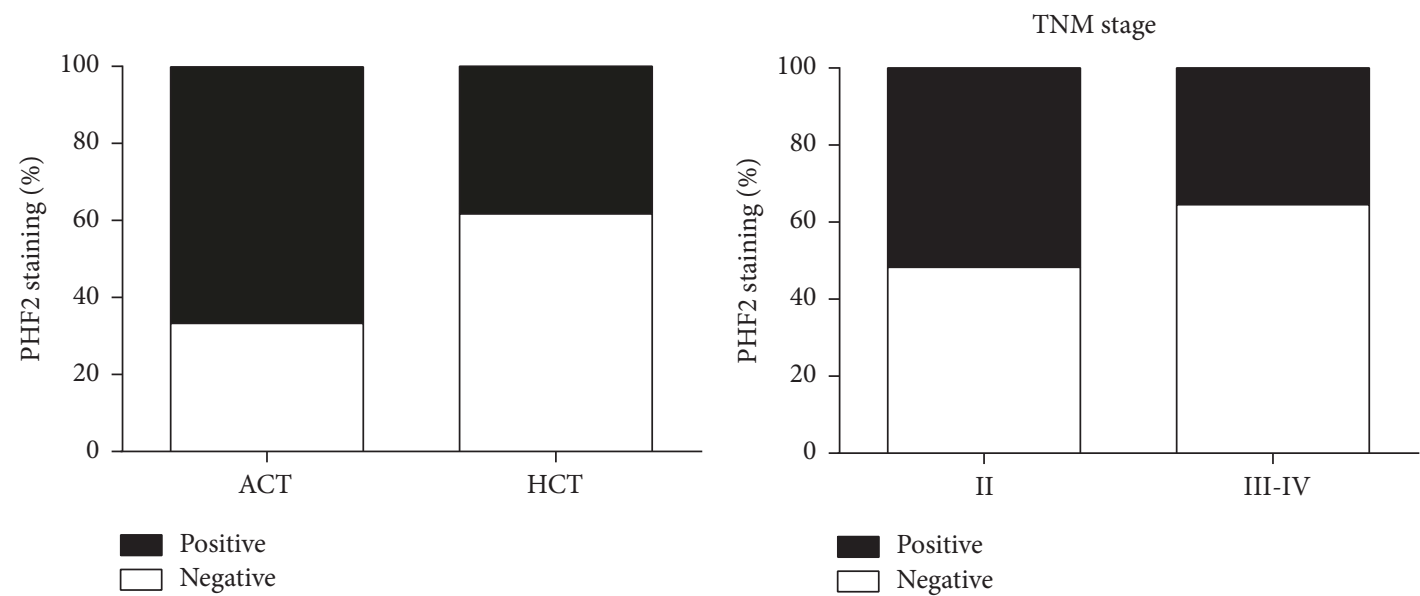

(b)

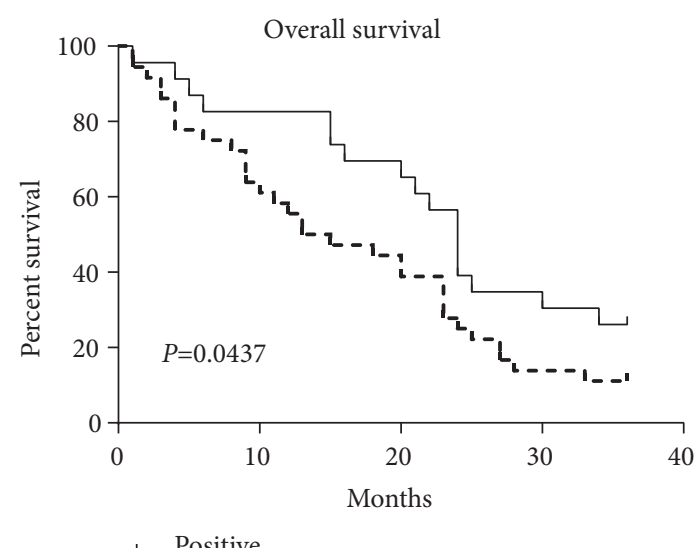

$\perp$ Positive

- เ. Negative

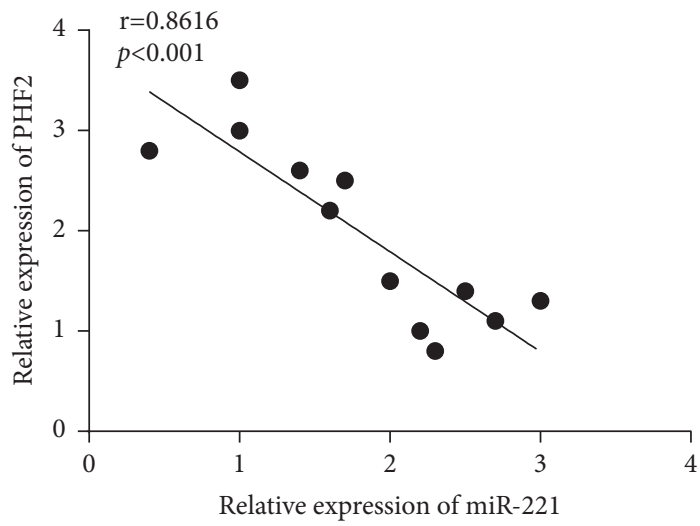

(d)

FIGURE 4: PHF2 is decreased in hepatocellular carcinoma tissues and associated with clinicopathological features in 60 hepatocellular cancer patients. (a) Representative photos of PHF2 expression patterns in hepatocellular tumors. The left panel depicts matched adjacent cancer tissues, whereas the right panel represents the hepatocellular carcinoma tissues (original magnification, $\times 100$ ). The magnifying detail of the immunohistochemical analysis for each case can be shown in the bottom side (original magnification, $\times 200$ ). (b) Compared with the adjacent cancer tissues, the overall expression level of PHF2 in the hepatocellular carcinoma tissues was significantly lower, P<0.05, $\chi 2$ test. PHF2 expression was correlated with TNM stage, $\mathrm{P}<0.05, \chi 2$ test. (c) Kaplan-Meier estimates of the probability of 3-year overall survival according to low and high PHF2 expression of 60 patients with hepatocellular cancer. $P=0.0437$, log-rank test. (d) The correlation between the protein expression of miR-221 and PHF2 was measured by Pearson's correlation coefficient analysis. 
TABLE 2: Patients' characteristics and PHF2 expression. $*$ P values are obtained from $\chi 2$ test.

\begin{tabular}{|c|c|c|c|c|}
\hline \multirow{2}{*}{ Variables } & \multicolumn{4}{|c|}{ PHF2 staining } \\
\hline & $\operatorname{High}(\%)$ & Low $(\%)$ & Total & $P *$ \\
\hline All points & $23(38.3)$ & $37(61.7)$ & 60 & \\
\hline \multicolumn{5}{|l|}{ Age } \\
\hline$\leq 57$ years & $5(33.3)$ & $10(66.7)$ & 15 & 0.646 \\
\hline$>57$ years & $18(40.0)$ & $27(60.0)$ & 45 & \\
\hline \multicolumn{5}{|l|}{ Gender } \\
\hline Male & $16(41.0)$ & $23(59.0)$ & 39 & 0.559 \\
\hline Female & $7(33.3)$ & $14(66.7)$ & 21 & \\
\hline \multicolumn{5}{|l|}{ Tumor size } \\
\hline$\leq 7 \mathrm{~cm}$ & $13(27.9)$ & $25(72.1)$ & 38 & 0.388 \\
\hline$>7 \mathrm{~cm}$ & $10(34.5)$ & $12(65.5)$ & 22 & \\
\hline \multicolumn{5}{|l|}{ pT status } \\
\hline $\mathrm{pT}_{1-2}$ & $8(61.5)$ & $5(38.5)$ & 13 & 0.013 \\
\hline $\mathrm{pT}_{3}$ & $12(46.2)$ & $14(53.8)$ & 26 & \\
\hline $\mathrm{pT}_{4}$ & $3(14.3)$ & $18(85.7)$ & 21 & \\
\hline \multicolumn{5}{|l|}{ pN status } \\
\hline $\mathrm{pN}_{0}$ & $10(58.8)$ & $7(41.2)$ & 17 & 0.032 \\
\hline $\mathrm{pN}_{1}$ & $8(44.4)$ & $10(55.6)$ & 18 & \\
\hline $\mathrm{pN}_{2}-\mathrm{pN}_{3}$ & $5(20.0)$ & $20(80.0)$ & 25 & \\
\hline \multicolumn{5}{|l|}{ TNM stage } \\
\hline II & $13(56.5)$ & $10(43.5)$ & 23 & 0.022 \\
\hline III-IV & $10(27.0)$ & $27(73.0)$ & 37 & \\
\hline \multicolumn{5}{|l|}{ Serum AFP } \\
\hline$\leq 400 \mu \mathrm{g} / \mathrm{L}$ & $14(53.8)$ & $12(46.2)$ & 26 & 0.031 \\
\hline$>400 \mu \mathrm{g} / \mathrm{L}$ & $9(26.5)$ & $25(73.5)$ & 34 & \\
\hline \multicolumn{5}{|c|}{ Microvascular invasion } \\
\hline Yes & $12(37.5)$ & $20(62.5)$ & 32 & 0.887 \\
\hline No & $11(39.3)$ & $17(60.7)$ & 28 & \\
\hline \multicolumn{5}{|c|}{ Portal vein tumor thrombus } \\
\hline Yes & $13(41.9)$ & $18(58.1)$ & 31 & 0.553 \\
\hline No & $10(34.5)$ & $19(65.5)$ & 29 & \\
\hline
\end{tabular}

cell migration and played its biological roles via regulating the PHF2 gene in HCC. Our characterization of this signaling pathway may provide novel therapeutic targets for the future treatment of HCC.

\section{Abbreviations}

miRNAs: MicroRNAs

PHF2: $\quad$ Plant homeodomain finger 2

HCC: Hepatocellular carcinoma

PHD: Plant homeodomain

TMA: Tissue microarray

HCT: Hepatocellular carcinoma tissues

EMT: Epithelial-to-mesenchymal transition

ESCC: Esophageal squamous cell carcinoma.

\section{Data Availability}

All relevant data used to support the findings of this study are included within the article.

\section{Conflicts of Interest}

The authors have declared that no conflicts of interest exist.

\section{Acknowledgments}

This work was supported by the project of Soochow Science and Technology Plan (SYS201676) and Natural Science Fund for Colleges and Universities in Jiangsu Province (09KJB310017).

\section{Supplementary Materials}

Supplemental Figure 1: quantitative PCR results of miR221 mRNA levels in HCC tissues (T) and in adjacent noncancerous tissues $(\mathrm{N})$. MiR-221 mRNA levels are higher in the HCC tissues (T) than in adjacent noncancerous tissues (N) $(n=24)$. Each bar represents the mean \pm SD of three independent experiments. $* * \mathrm{P}<0.01$. Supplemental Figure 2 : western blot was used to detect Snail and Slug proteins level 
in miR-221-overexpression and miR-221-knockdown SMMC7721 cells. Supplemental Figure 3: the histograms that express grayscale of Figures $2 \mathrm{C}-\mathrm{D}$. Each bar represents the mean \pm SD of three independent experiments. $* * \mathrm{P}<0.01$. Supplemental Figure 4: qRT-PCR results of PHF2 mRNA levels in HCC cell lines and human normal hepatocyte. Each bar represents the mean $\pm \mathrm{SD}$ of three independent experiments. $* * \mathrm{P}<0.01$. (Supplementary Materials)

\section{References}

[1] W. Chen, R. Zheng, P. D. Baade et al., "Cancer statistics in China, 2015," CA: A Cancer Journal for Clinicians, vol. 66, pp. 115-132, 2016.

[2] H. B. El-Serag and K. L. Rudolph, "Hepatocellular carcinoma: epidemiology and molecular carcinogenesis," Gastroenterology, vol. 132, no. 7, pp. 2557-2576, 2007.

[3] D. P. Bartel, "MicroRNAs: genomics, biogenesis, mechanism, and function," Cell, vol. 116, no. 2, pp. 281-297, 2004.

[4] S. L. Ameres and P. D. Zamore, "Diversifying microRNA sequence and function," Nature Reviews Molecular Cell Biology, vol. 14, no. 8, pp. 475-488, 2013.

[5] Y.-J. Pan, L.-L. Wei, X.-J. Wu, F.-C. Huo, J. Mou, and D.-S. Pei, "MiR-106a-5p inhibits the cell migration and invasion of renal cell carcinoma through targeting PAK5," Cell death \& Disease, vol. 8, no. 10, Article ID e3155, 2017.

[6] C. S.-C. Lam, L. Ng, A. K.-M. Chow et al., "Identification of microRNA 885-5p as a novel regulator of tumor metastasis by targeting CPEB2 in colorectal cancer," Oncotarget, vol. 8, pp. 26858-26870, 2017.

[7] B. Ell, L. Mercatali, T. Ibrahim et al., "Tumor-induced osteoclast miRNA changes as regulators and biomarkers of osteolytic bone metastasis," Cancer Cell, vol. 24, no. 4, pp. 542-556, 2013.

[8] M. Garofalo, C. Quintavalle, G. Romano, C. M. Croce, and G. Condorelli, "miR221/222 in cancer: their role in tumor progression and response to therapy," Current Molecular Medicine, vol. 12, no. 1, pp. 27-33, 2012.

[9] Q. Wu, X. Ren, Y. Zhang et al., "MiR-221-3p targets ARF4 and inhibits the proliferation and migration of epithelial ovarian cancer cells," Biochemical and Biophysical Research Communications, vol. 497, no. 4, pp. 1162-1170, 2018.

[10] K. Hasenpusch-Theil, B. P. Chadwick, T. Theil, S. K. Heath, D. G. Wilkinson, and A.-M. Frischauf, "PHF2, a novel PHD finger gene located on human Chromosome 9q22," Mammalian Genome, vol. 10, no. 3, pp. 294-298, 1999.

[11] K. Lee, U. Ju, J. Song, and Y. Chun, “The histone demethylase PHF2 promotes fat cell differentiation as an epigenetic activator of both C/EBPalpha and C/EBPdelta," Molecules and Cells, vol. 37, pp. 734-741, 2014.

[12] K.-H. Lee, J.-W. Park, H.-S. Sung et al., "PHF2 histone demethylase acts as a tumor suppressor in association with p53 in cancer," Oncogene, vol. 34, no. 22, pp. 2897-2909, 2015.

[13] Y. Li, Z. Ren, Y. Wang et al., "ADAM17 promotes cell migration and invasion through the integrin betal pathway in hepatocellular carcinoma," Experimental Cell Research, 2018.

[14] Y. Zhou, P. He, X. Xie, and C. Sun, "Knockdown of SUMO1P3 represses tumor growth and invasion and enhances radiosensitivity in hepatocellular carcinoma," Molecular and Cellular Biochemistry, 2018.

[15] H. Byeon, S. D. Lee, E.-K. Hong et al., "Long-term prognostic impact of osteopontin and Dickkopf-related protein 1 in patients with hepatocellular carcinoma after hepatectomy," Pathology - Research and Practice, vol. 214, no. 6, pp. 814-820, 2018.

[16] F. Vasuri, M. Visani, G. Acquaviva et al., "Role of microRNAs in the main molecular pathways of hepatocellular carcinoma," World Journal of Gastroenterology, vol. 24, no. 25, pp. 26472660, 2018.

[17] J. Quan, Y. Li, X. Pan et al., "Oncogenic miR-425-5p is associated with cellular migration, proliferation and apoptosis in renal cell carcinoma," Oncology Letters, vol. 16, pp. 2175-2184, 2018.

[18] E. N. Howe, D. R. Cochrane, and J. K. Richer, “The miR-200 and miR-221/222 microRNA families: opposing effects on epithelial identity," Journal of Mammary Gland Biology and Neoplasia, vol. 17, no. 1, pp. 65-77, 2012.

[19] E. Lambertini, A. Lolli, F. Vezzali, L. Penolazzi, R. Gambari, and R. Piva, "Correlation between Slug transcription factor and miR-221 in MDA-MB-231 breast cancer cells," BMC Cancer, vol. 12, p. 445, 2012.

[20] C. Le Sage, R. Nagel, D. A. Egan et al., "Regulation of the p27(Kip1) tumor suppressor by miR-221 and miR-222 promotes cancer cell proliferation," EMBO Journal, vol. 26, no. 15, pp. 3699-3708, 2007.

[21] F. Fornari, L. Gramantieri, M. Ferracin et al., "MiR-221 controls $\mathrm{CDKN1C/p57} \mathrm{and} \mathrm{CDKN1B/p27} \mathrm{expression} \mathrm{in} \mathrm{human} \mathrm{hepato-}$ cellular carcinoma," Oncogene, vol. 27, no. 43, pp. 5651-5661, 2008.

[22] M. Singla and S. Bhattacharyya, "Autophagy as a potential therapeutic target during epithelial to mesenchymal transition in renal cell carcinoma: An in vitro study," Biomedicine \& Pharmacotherapy, vol. 94, pp. 332-340, 2017.

[23] I. K. Guttilla, K. N. Phoenix, X. Hong, J. S. Tirnauer, K. P. Claffey, and B. A. White, "Prolonged mammosphere culture of MCF-7 cells induces an EMT and repression of the estrogen receptor by microRNAs," Breast Cancer Research and Treatment, vol. 132, no. 1, pp. 75-85, 2012.

[24] Editors PO, "Retraction: miR-221 Promotes Tumorigenesis in Human Triple Negative Breast Cancer Cells," PLoS One, vol. 12, Article ID e0175869, 2017.

[25] C. Lee, B. Kim, B. Song, and K. C. Moon, "Implication of PHF2 expression in clear cell renal cell carcinoma," Journal of Pathology and Translational Medicine, vol. 51, no. 4, pp. 359364, 2017.

[26] L.-L. Sun, X.-X. Sun, X.-E. Xu et al., “Overexpression of Jumonji AT-rich interactive domain $1 \mathrm{~B}$ and $\mathrm{PHD}$ finger protein 2 is involved in the progression of esophageal squamous cell carcinoma," Acta Histochemica, vol. 115, no. 1, pp. 56-62, 2013. 


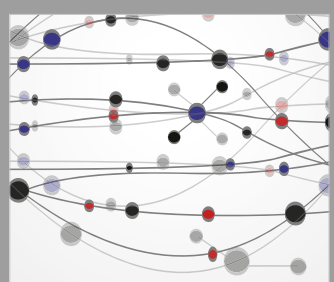

The Scientific World Journal
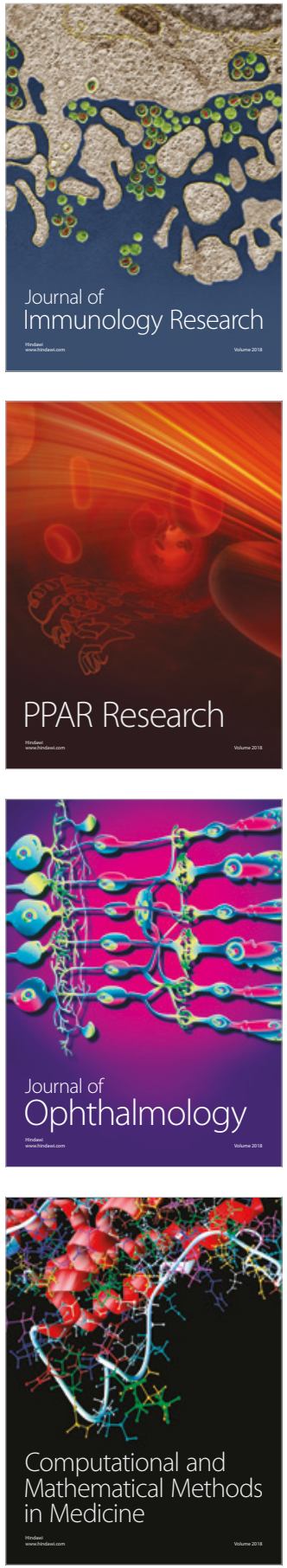

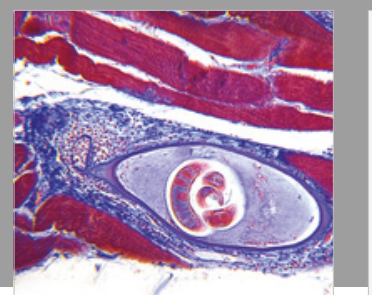

Gastroenterology Research and Practice

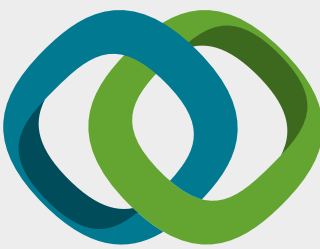

\section{Hindawi}

Submit your manuscripts at

www.hindawi.com
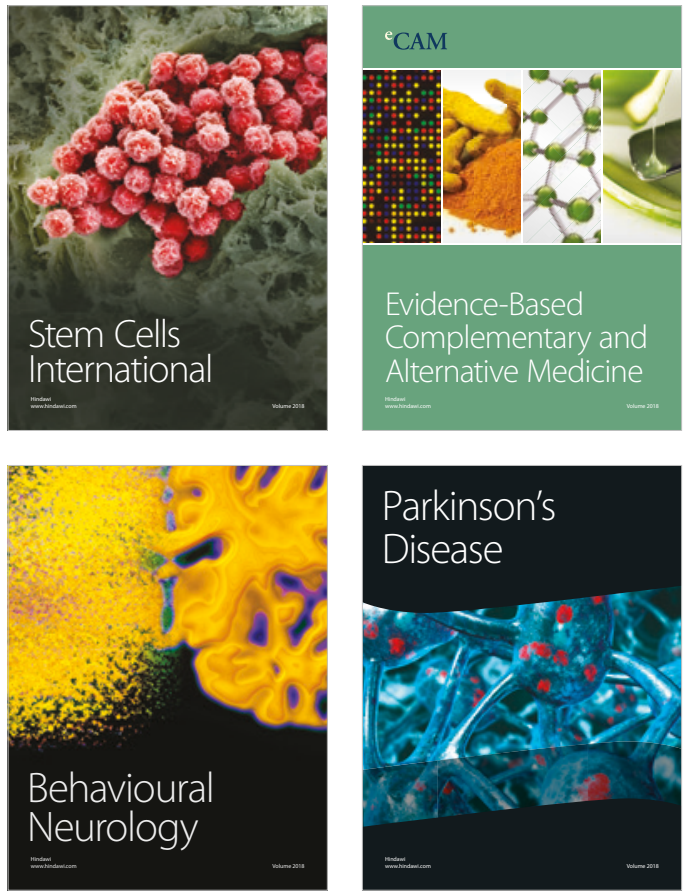

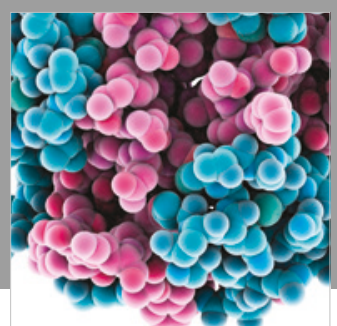

ournal of

Diabetes Research

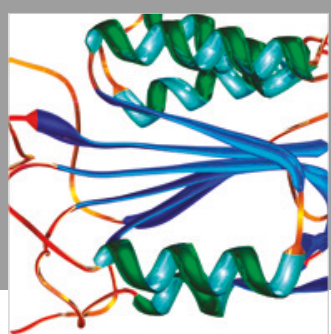

Disease Markers
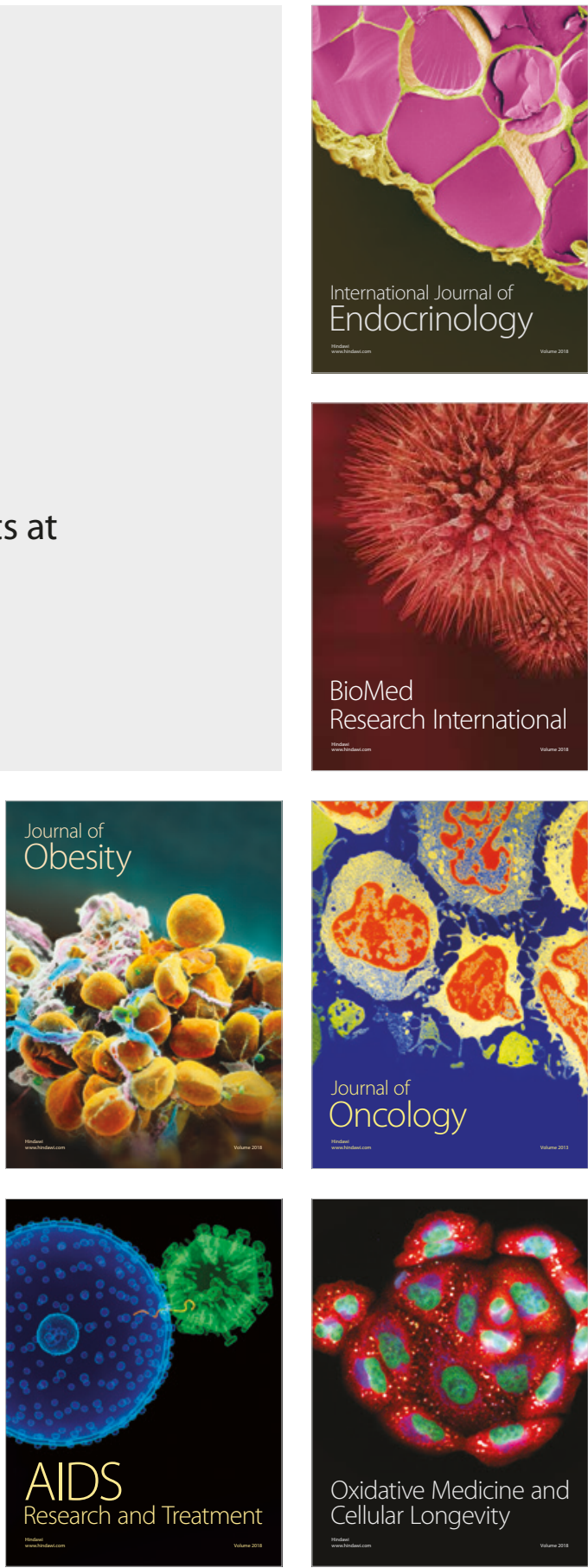